ANL/MSD/CP--87985
CONF-9510119--28

\title{
Why Cryogenically Cooled, Thin Cyrstals Handle Extremely High Power Densities
}

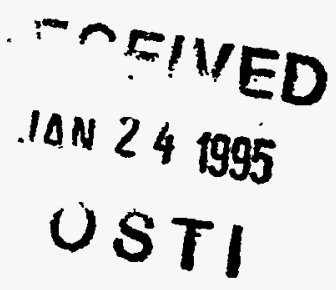

G.S. Knapp, G. Jennings and $M . A$. Beno

Materials Sclence Division. Argonne National Laboratory, Argonne, IL 6043

Materials Science Division

Argonne National Laboratory

Argonne, IL 60439

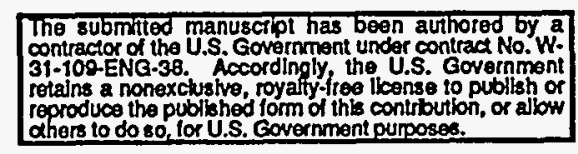

September 1995

\section{DISCLAIMER}

/je

\section{Distribution:}

1-2. M. J. Masek

3. B. D. Dunlap

4. P. A. Montano

5. F. Y. Fradin

6. R. Gottschall .

7. Editorial Office

8. Authors
This report was prepared as an account of work sponsored by an agency of the United States Government. Neither the United States Government nor any agency thereof, nor any of their employees, makes any warranty, express or implied, or assumes any legal liability or responsibility for the accuracy, completeness, or usefulness of any information, apparatus, product, or process disclosed, or represents that its use would not infringe privately owned rights. Reference herein to any specific commercial product, process, or service by trade name, trademark, manufacturer, or otherwise does not necessarily constitute or imply its endorsement, recommendation, or favoring by the United States Government or any agency thereof. The views and opinions of authors expressed herein do not necessarily state or reflect those of the United States Government or any agency thereof.

Submitted to the proceedings of the Synchrotron Radiation Instrumentation '95 conference to be published as a supplement to the Review of Scientific Instruments.

This work is supported by the Division of Materials Sciences, Office of Basic Energy Sciences of DOE, under contract No. W-31-109-ENG-38. 


\title{
Why Cryogenically Cooled,Thin Crystal Handle Extremely High Power Densities
}

G. S. Knapp, G. Jennings and M. A. Beno Materials Science Division, Argonne

National Laboratory, Argonne IL 60439

\begin{abstract}
Recently, a new type of cryogenically cooled high heat load monochromator was proposed and 1 , developed at Argonne National Laboratory ${ }^{2}$ and tested ${ }^{3}$ at European Synchrotron Radiation Facility (ESRF.) These tests showed that powers of $153 \mathrm{~W}$ and power densities of $450 \mathrm{~W} / \mathrm{mm}^{2}$ cause only negligible strain. These powers and power densities are larger than will be absorbed by the first crystal on an undulator beamline at the Advanced Photon Source (APS ). In our earlier work we suggested that the crystal might show strain at much lower values of the powers and power densities. We now can explain the ESRF results in terms of the unique role the negative thermal expansion coefficient of Si plays in minimizing strain.
\end{abstract}


Earlier work $1,2,3$, showed that a cryogenically cooled monochromator employing a thin channel machined in a large cooled block of Si crystal, is capable of handling the high heat loads to be generated by the Advanced Photon Source (APS). The thin section absorbs only a fraction of the incident beam power when the undulator is operating at closed gap. The fact that the crystal is thin combined with the high thermal conductivity and the negative or very small thermal expansion at low temperatures causes the crystal surface to be under tension and therefore will not buckle. Another important factor is that the thermal conductivity of Si at liquidnitrogen temperatures is so high that the $\mathrm{Si}$ can be cooled with the cooling surfaces at some reasonable distance from the thin part of the crystal thereby allowing the heat to spread out. This overcomes the fact that the heat transfer coefficient of liquid nitrogen is quite small, hence requiring large surface areas to transfer the heat. Figure 1 shows a drawing of the design for the first crystal of a monochromator for undulator A. Two narrow vee shaped channels, with a minimum width of $2.4 \mathrm{~mm}$ wide, are machined into opposite sides of a Si block. The thin, center strip of Si which diffracts the incident beam is approximately $15 \mathrm{~mm}$ long and $0.6 \mathrm{~mm}$ thick (the vee shape is used because alignment ${ }^{3}$ of the crystal direction was difficult). For clarity we show only one half of the $\mathrm{Si}$ crystal block. The crystal strip will be reasonably strain free because of the crystals rectangular shape with slots to let the beam miss the thick sections of the crystal over the range of angles $5^{\circ}$ to $32^{\circ}$. Invar blocks are clamped to the ends of the crystal via In gaskets and the liquid nitrogen is pumped through the holes in the Si.

In order to test how the thin cryogenically cooled crystal will work at APS, it was necessary to perform tests at a high power beamline. Such a facility was available at ESRF using a focused wiggler. The experiments ${ }^{3}$ were performed at ESRF BL3 at about $15 \mathrm{~m}$ from a Toroidal mirror which produced a focal spot $.2 \mathrm{~mm}$ high by $1.8 \mathrm{~mm}$ wide. The maximum power delivered to the sample was $167 \mathrm{~W}$ of which $154 \mathrm{~W}$ were absorbed by the crystal. This power and power density was much in excess of that expected at APS. The beam hit the crystal at its center and the Bragg angle was $11.4^{\circ}$. The $\mathrm{Si}(333)$ reflection was monitored $(30 \mathrm{KeV})$ with a matching $\mathrm{Si}(333)$ monochromator. There was mounting strain of order 2 arcseconds in these tests (the Darwin width of this reflection is 0.5 arcsec). The double crystal rocking curves are shown in figures 2 and3. 
Figure 2 shows the results of a measurement done at room temperature with 24 $\mathrm{W}$ of absorbed power. Note the large broading of the rocking curve. In figure 4 we show the rocking cures of the cryogenically cooled monochromator. Note that as a function of power the widths narrow slightly.

In the ealier work ${ }^{1,2}$ the assumption was made that as long as the temperature stayed below about $120 \mathrm{~K}$, i.e. in the negative thermal expansion region, there would be no bowing. The maximum calculated absorbed power was less than the power used in the ESRF experiments and the power densities were much less. Why did the ESRF experiment not show strain? These results can be explained if one carries out an approximate thermal analysis of the ESRF results. Yang et al ${ }^{4}$ first showed how the temperature profile of the $\mathrm{Si}$ could be calculated in the earlier work ${ }^{1,2}$ we made the assumption that as long as the peak temn a similar geometry if the temperature dependence of the $\mathrm{Si}$ was approximated by the following equation.

$$
\kappa(T)=\frac{\Gamma}{\left(T-T_{\kappa}\right)}
$$

where $\Gamma=446.3 \mathrm{~W} / \mathrm{cm}$ and $T_{K}=47.4 \mathrm{~K}$. Following Yang et al . 4 , the temperature profile across the strip can be calculated 1 from

$$
T(x)=T_{K}+\left(T_{0}-T_{K}\right) \exp \left[\frac{q}{2 \Gamma}\left(\frac{w^{2}}{4}-x^{2}\right)\right],
$$

where $T_{0}$ is the temperature at the edge of the strip, $w$ is the width of the strip, and $x$ is the distance from the center line of the strip and is less than or equal to $w / 2$. In this paper we use this equation and the other approximate calculations 1 to calculate the temperature distribution across the strip using the parameters appropriate to the ESRF experiment. These approximations have been shown to give accurate results by comparison with finite element results. We also need to approximate the temperature profile in the other direction along the center line of the beam. In this direction we assume the profile will be gaussian with a width approximately the width of the beam footprint. (We shall show below that the results for thermal strain do not depend strongly on this assumption.) 
Next we need to this the thermal strain resulting from this temperature profile. Again we follow Yang, Meron, Ruan, and Schildkamp 4 who discussed much of the same physics as applied to the cryogenic silicon Laue monochromator. They showed that because of the region of negative thermal expansion of Si below 120 $\mathrm{K}$, a thin strip, such as we have here, will be under tension as it first heats up, and because of this tension there will be no bowing of the strip. This thermal contraction can actually flatted the strip if it is slightly bowed due to mounting strain. In our earlier work we set as a limit that the peak temperature in the center of the strip should be near the point where the thermal expansion goes to zero. However, as Yang et al ${ }^{4}$ show, until a volume element of Si reaches a much higher temperature, this element will still be smaller than it was before it is heated. This temperature is about $160 \mathrm{~K}$. Even if the peak temperature is larger than this, the cooler sections will still be contracted so the strip will be under tension. The equation for the total expansion of a length of crystal is;

$$
\frac{\Delta l}{l}=\frac{\int d y \int_{T_{0}}^{T(y)} \alpha\left(T^{\prime}\right) d T^{\prime}}{\int d y}
$$

where $\frac{\Delta l}{l}$ is the fractional expansion( or contraction) along the $y$ direction, $T(y)$ is the peak temperature, $T_{0}$ is the minimum temperature, and $\alpha(T)$ is the thermal expansion coefficient which Yang et al ${ }^{4}$ parameterized by $\alpha(T)=A\left(T-T_{\alpha}\right)$ where $T_{\alpha}=120.5 K$ and $A=1.79 \times 10^{-6}\left(K^{-2}\right)$. We have approximated the temperature profile across the center line of the strip (transverse direction) by eq. 2 . Below we show the peak temperature of the strip has a function of power using the parameters which are appropriate for the ESRF experiments.

We also need to examine the expansion in the longitudinal direction. We have no analytical solution for the heat flow in this direction but it is clear that it will be roughly gaussian with a width approximately the same as the beam footprint. We have varied this width from 1 to $2 \mathrm{~mm}$ and found the fractional expansion to be reasonably insensitive to the model. In figure 5 we show $\frac{\Delta l}{l}$ in longitudinal 
and transverse directions. At power levels where $\frac{\Delta l}{l}$ is negative there will be no bowing, if anything the strip should flatten, and that seems to be what is happening in the ESRF experiments ( figure 2).

\section{Acknowledgments}

This work would not have possible without the work of the C. S. Rogers and D. M. Mills who were instrumental in developing the cryogenic crystal from a concept into a reality. The team of experimntalists ${ }^{3}$ that participated in the ESRF experiments did a fantastic job. It is as a result of these experiments that most of the beamlines at APS will use a thin cryogenic crystal as the first optical element.

\section{References}

1. G. S. Knapp, M. A. Beno, C. S. Rogers, C. L. Wiley, P. A. Cowan, Rev. Sci.. Instrum. 65, 2792 (1994)

2. G. S. Knapp, C. S. Rogers, M. A. Beno, G. Jennings, P. A. Cowan, Rev. Sci.. Instrum. 66, 2138 (1995)

3. C. S. Rogers, D. M. Mills, W. K. Lee, G. S. Knapp, J. Holmberg, A. Freund, M. Wulff, M. Rossat, M Hanfland, H, Yamaoka,Rev. Sci.. Instrum. 66, 3494 (1995) 4. B. X. Yang, M. Meron, Y. Ruan, and W. Schildkamp. Pro. Soc. of Photo-Optical Instrum. Eng. 1997,302, 1993 


\section{Figure Captions}

Fig. 1 Cross-section schematic of the cryogenically cooled, thin crystal silicon monochromator showing the incident, diffracted and transmitted beams.

Fig. $2 \mathrm{Si}(333)$ double-crystal rocking curve for the uncooled, room-temperature crystal with $24 \mathrm{~W}$ absorbed.

Fig. $3 \mathrm{Si}(333)$ double-crystal rocking curves for the cryogenically cooled monochromator for several absorbed powers (the curves are displaced along both axes for clarity).

Fig. 4 The Calculated peak temperatures versus power for the ESRF experiment

Fig. 5 The Calculated Thermal expansions in the longitudinal and transverse Directions versus power for the ESRF experiment. 


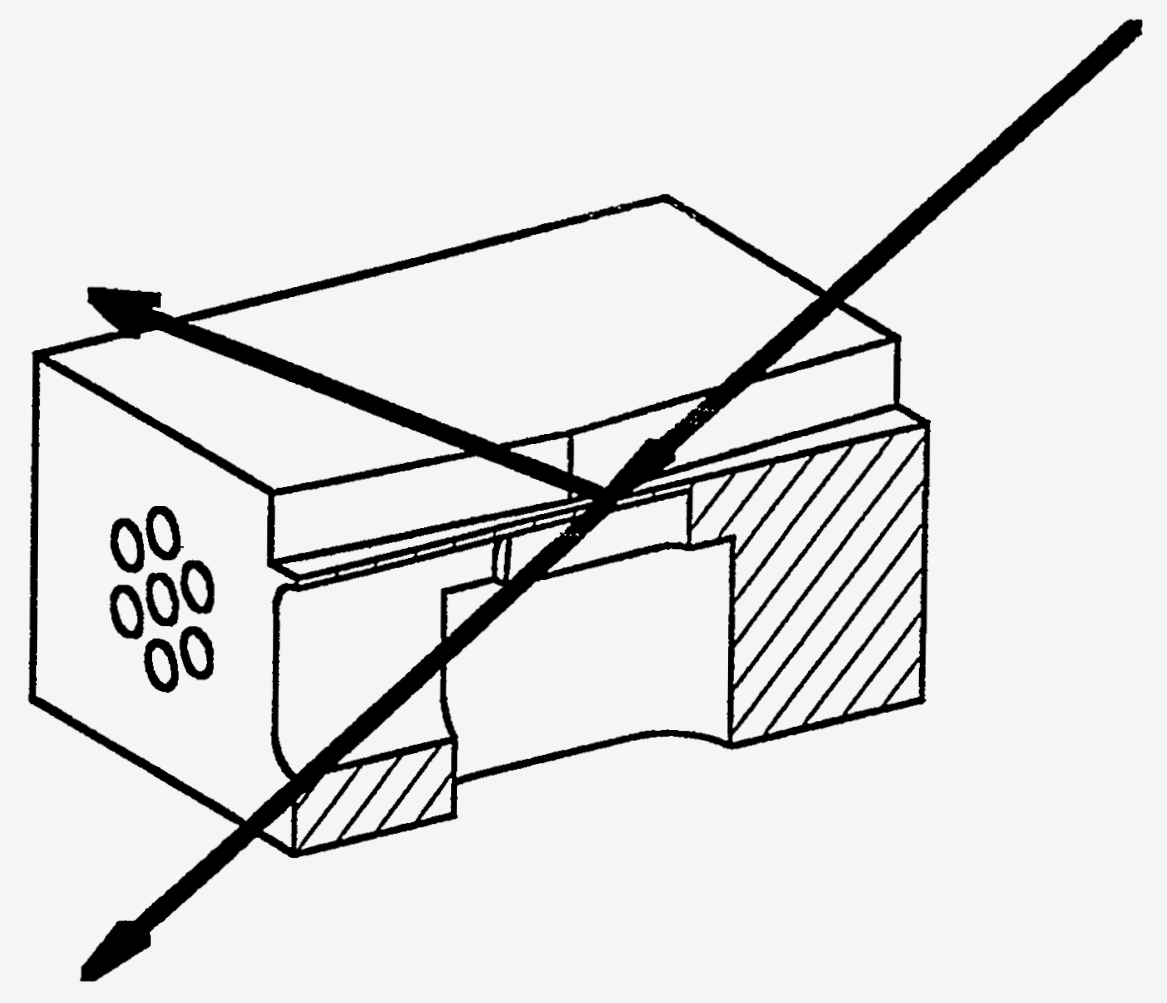

Fig. 1 


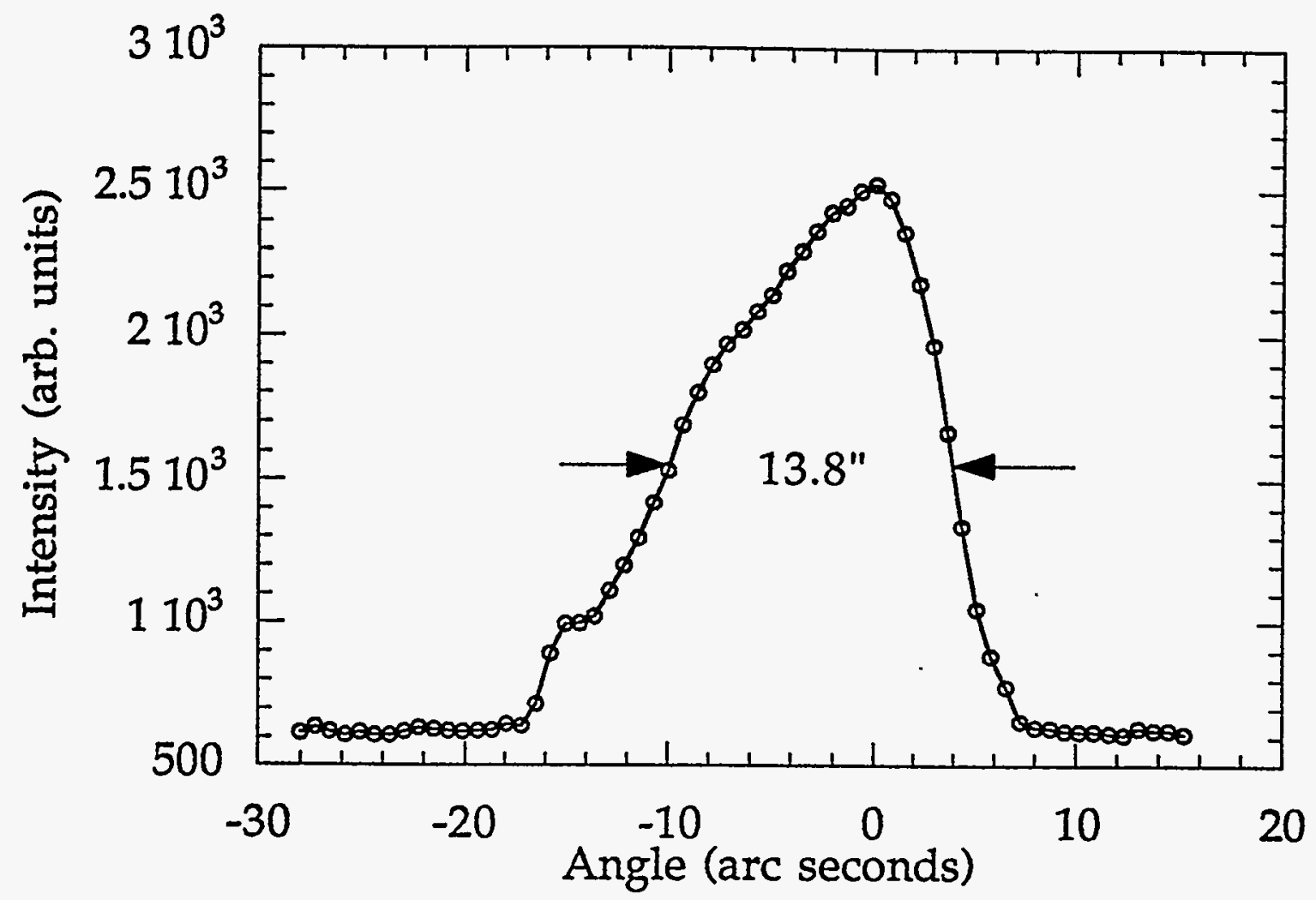

Fig. 2 


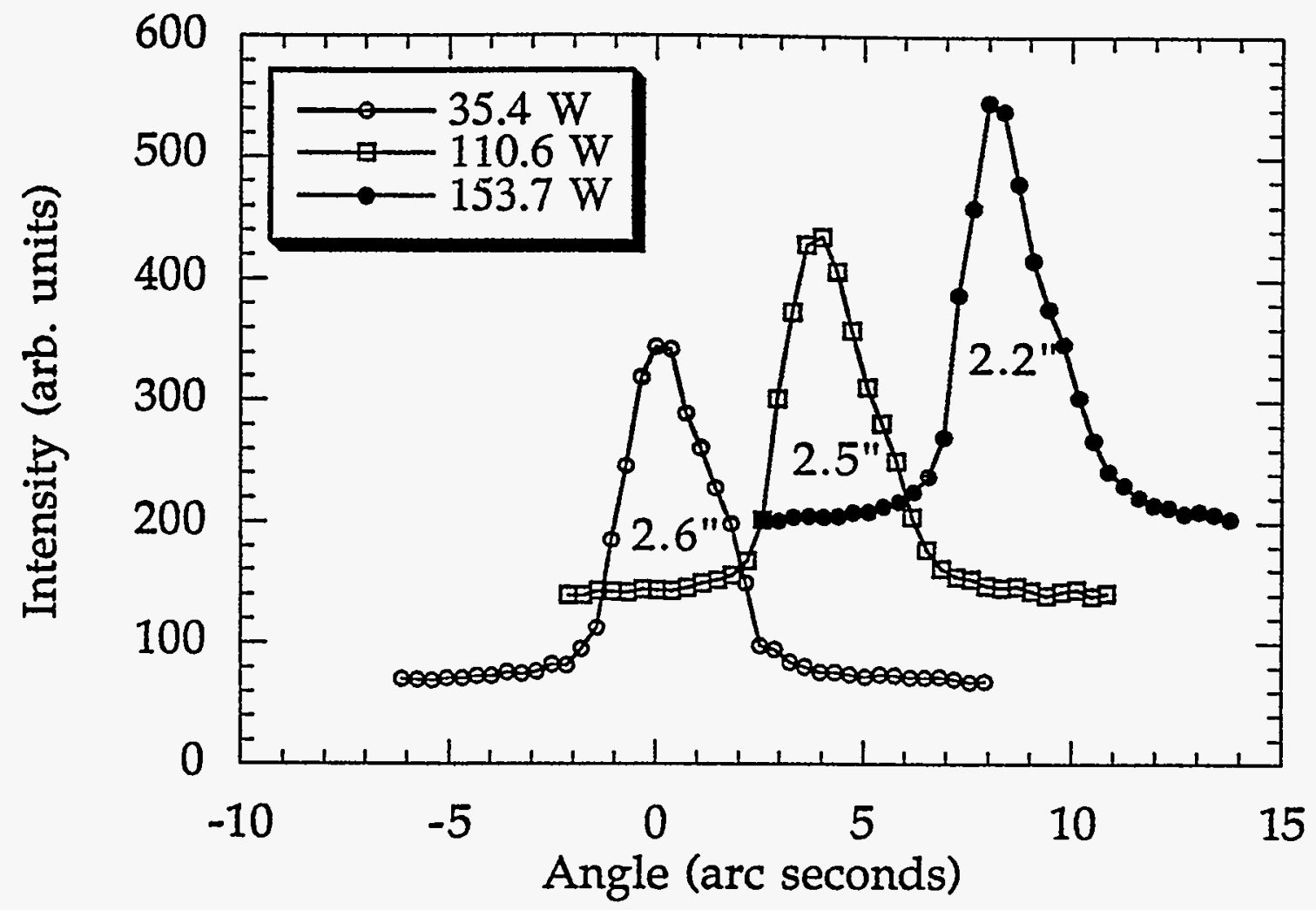

Fig 3 


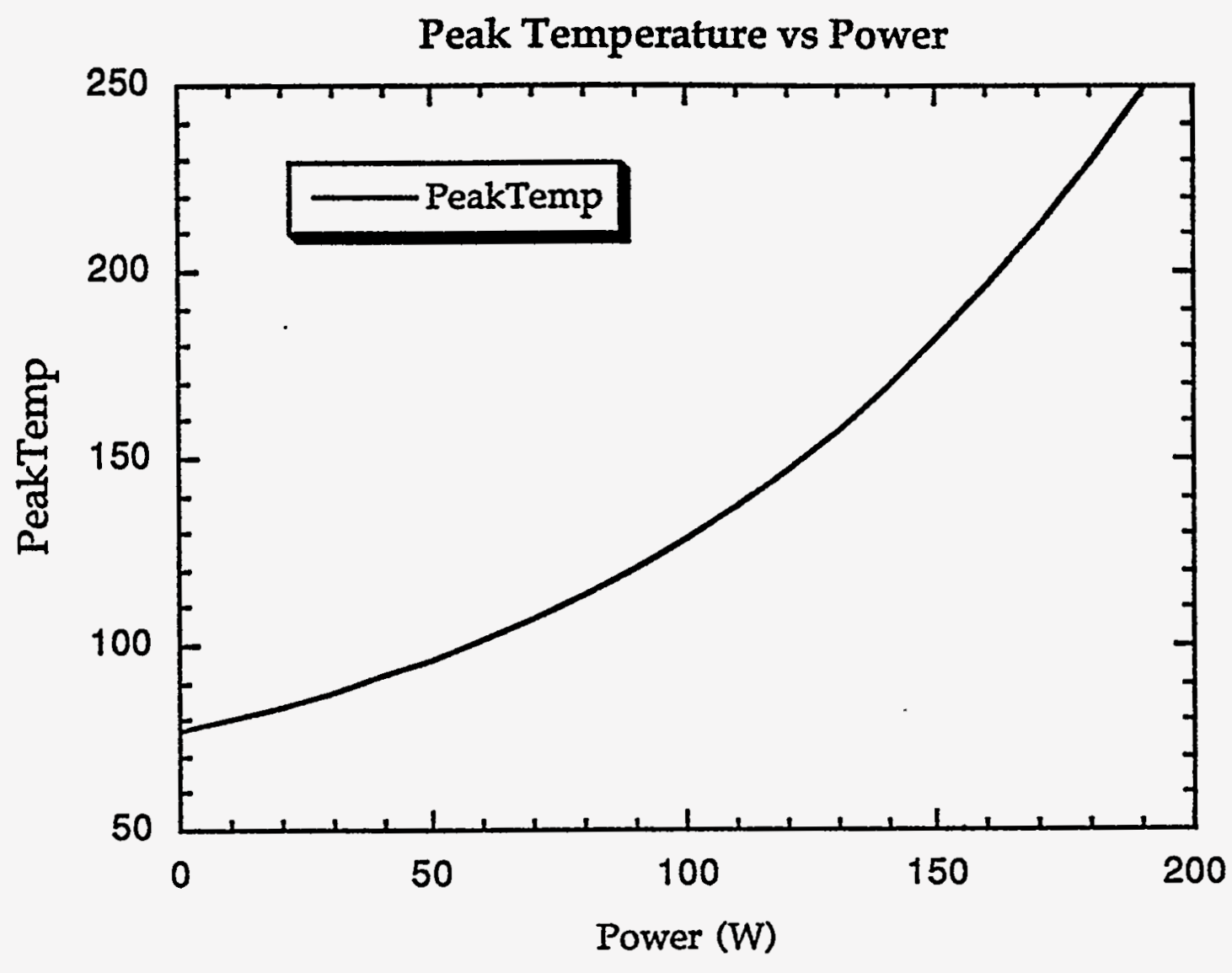

fig 4 


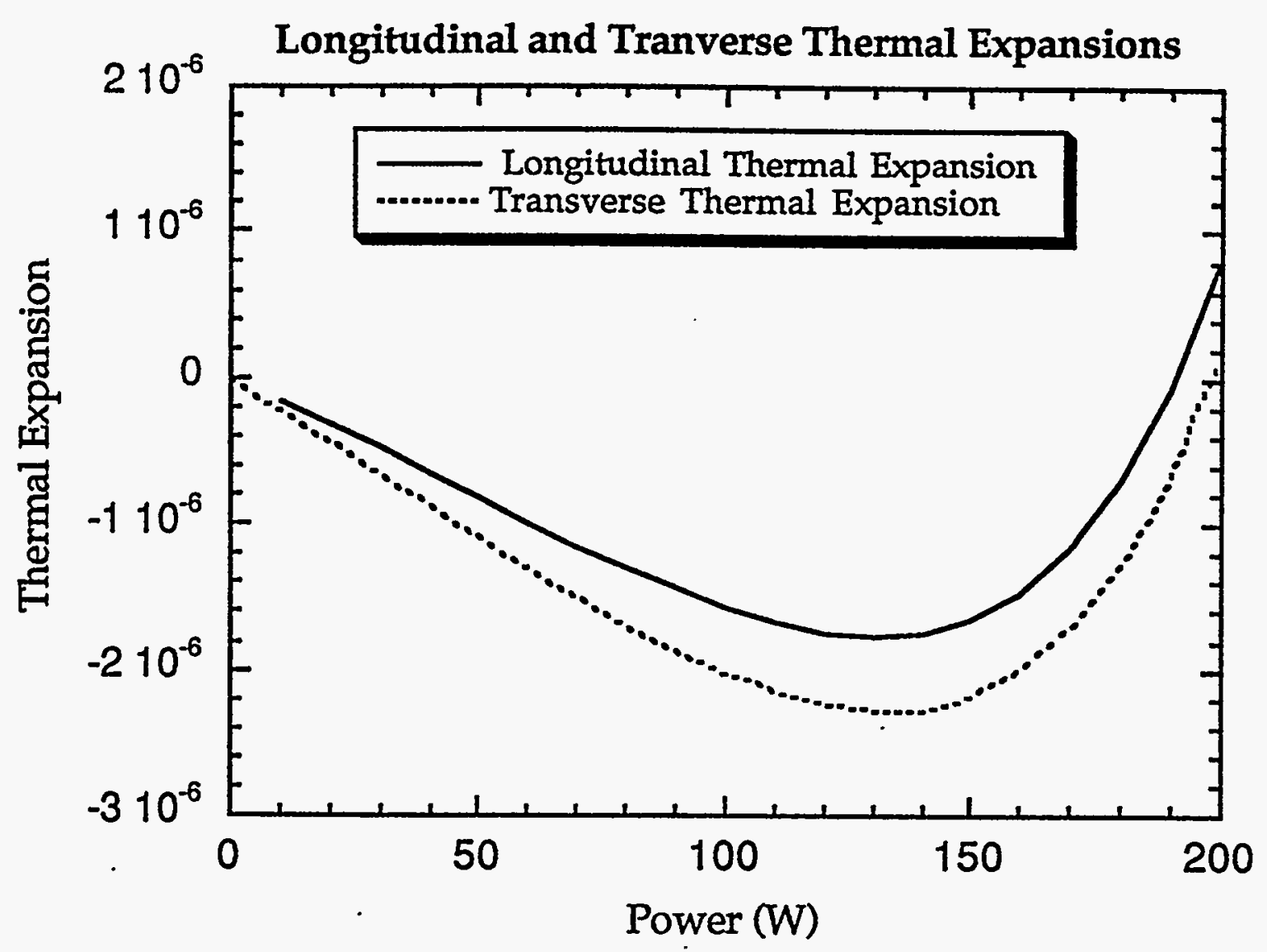

Fig5 